International Journal of Bifurcation and Chaos, Vol. 15, No. 9 (2005) 2783-2798

(C) World Scientific Publishing Company

\title{
DELAY, PARAMETRIC EXCITATION, AND THE NONLINEAR DYNAMICS OF CUTTING PROCESSES
}

\author{
GÁBOR STÉPÁN*, TAMÁS INSPERGER ${ }^{\dagger}$ and RÓBERT SZALAI ${ }^{\ddagger}$ \\ Department of Applied Mechanics, \\ Budapest University of Technology and Economics, \\ Budapest, H-1521, Hungary \\ *stepan@mm.bme.hu \\ †inspi@mm.bme.hu \\ ${ }^{\ddagger}$ szalai@mm.bme.hu
}

Received February 22, 2005

\begin{abstract}
It is a rule of thumb that time delay tends to destabilize any dynamical system. This is not true, however, in the case of delayed oscillators, which serve as mechanical models for several surprising physical phenomena. Parametric excitation of oscillatory systems also exhibits stability properties sometimes defying our physical sense. The combination of the two effects leads to challenging tasks when nonlinear dynamic behaviors in these systems are to be predicted or explained as well. This paper gives a brief historical review of the development of stability analysis in these systems, induced by newer and newer models in several fields of engineering. Local and global nonlinear behavior is also discussed in the case of the most typical parametrically excited delayed oscillator, a recent model of cutting applied to the study of high-speed milling processes.
\end{abstract}

Keywords: Time delay; time periodic systems; regenerative effect; cutting.

\section{Introduction}

One of the most challenging tasks in engineering is the stabilization of oscillatory systems with small damping and large time delay. Although the earliest examples of this category of problems were published in biomathematics (see the predator-prey model of [Volterra, 1928]), the classical examples of such systems appeared not much later: the shimmying wheels with elastic tyres [Schlippe \& Dietrich, 1941; Stépán, 1998], the famous ship stabilization problem [Minorsky, 1942], then the material forming processes, like machine tool vibrations in the presence of the so-called regenerative effect [Tobias, 1965; Tlusty et al., 1962; Stépán, 1989]. Similar problems rose later during position and force control of robotic structures [Whitney, 1977; Raibert
\& Craig, 1981] in the presence of long time delay in the information transmission system [Stépán et al., 1990; Stépán, 2001a], in haptic control [Craig, 1986], or in flow separation point control [Insperger et al., 2004b]. One of the latest fields where the stability properties and nonlinear dynamics of delayed oscillators turn out to be essential is the neural network dynamics (see, for example, [Campbell, 1999]).

The corresponding mathematical theory of time delayed systems started developing in the early 1950's only [Myshkis, 1949; Bellman \& Cooke, 1963; Halanay, 1966; Hale \& Lunel, 1993; Diekmann et al., 1995], and consequently, the first algorithms for stability analyses and the resulting stability charts of the second-order autonomous systems showed up somewhat later in the works of Bhatt and Hsu

*Author for correspondence. 
[1966], Kolmanovskii and Nosov [1986], Stépán [1989], Niculescu [2000], Olgac and Sipahi [2002], $\mathrm{Hu}$ and Wang [2002], Insperger and Stépán [2004a], or Elbeyli and Sun [2004].

The idea and mathematical description of parametric excitation is older than that of the time delay. It goes back to the mid 19th century, when Mathieu [1868] derived his famous scalar second-order time-periodic differential equation to study the vibrations of elliptic membranes. This equation became later the mathematical model of the deeply studied pendulum with a periodically moving pivot.

The Floquet Theory going back also to the 19th century [Floquet, 1883] was developed for finite dimensional time-periodic systems (no time delays at that time, of course). This served as a theoretical basis for stability analysis without providing an actual algorithm. This is why the first stability results appeared in the literature only decades later, like the stabilization of the inverted pendulum by vibrating its pivot point vertically at a specific frequency [Stephenson, 1908], or the stability chart of the Mathieu equation [van der Pol \& Strutt, 1928; Ince, 1956]. The explanation of how the children's favorite toy, the swing works, was also quite late for the same reason [Levi \& Broer, 1995].

Nowadays, as both autonomous delayed oscillators and parametrically excited oscillators are fairly well understood, those engineering models appear and have come into focus, so the two effects may exist together. Of course, similar combination of models appear with stochastic systems (see, for example, [Elbeyli et al., 2005]) or with distributed parameter systems (see, for example, [Haller, 2004]), too, but the present study concentrates on delay and time-periodicity together. Such problems appeared in remote periodic motion and haptic control [Insperger \& Stepan, 2004d], or in control of periodic flows, but one of the most transparent engineering problems is high-speed milling in this respect.

The industrial success of high-speed milling has directed research efforts of experts in the field of machine tool vibrations to the understanding of the peculiar stability properties of the so-called highly interrupted cutting. Without going into the details of all the complex modeling issues of high-speed milling, it is worthwhile to understand the basic physical phenomena since it represents well the central problems of time-periodic and time-delayed systems.
Regenerative effect in material cutting is a kind of self-excited vibration. The tool supported by an elastic structure cuts the wavy surface of the rotating workpiece. This wavy surface is actually formed by the vibration of the tool itself one period earlier. Through the variation of the chip thickness, a varying cutting force acts on the tool, and this variation of force depends on the past oscillation of the tool. There is a long time delay in this oscillatory system, which is inversely proportional to the cutting speed.

The above scenario is valid for conventional turning, where the parameters are constant. In case of high-speed milling, the thin finger-like tool is rotating, and the workpiece is steady. The tool has low number of cutting edges, typically only 2 . During operation, the cutting force has a strong timeperiodic character caused by the periodic variation of the contact between the workpiece and the tool. In extreme cases, the time spent from cutting to not cutting is below 0.1, that is, the cutting edges fly over the surface of the workpiece without contact most of the time, and there are only short time intervals when one of the edges hits the workpiece and removes chip. The regenerative effect, however, still exists during these contact periods (see the models of Minis and Yanushevsky [1993], Altintas and Budak [1995], Balachandran [2001], Gouskov et al. [2002], Corpus and Endres [2004], Faassen et al. [2004], Peigne et al. [2004]). Actually, the time period is just equal to the time delay in these models.

The great efficiency of stable high-speed milling in material formation represents an excellent possibility of stabilizing a delay-system with parametric excitation. One of the direct applications of this idea is the stabilization of the cutting process via spindle speed variation [Inamura \& Sata, 1974; Takemura et al., 1974; Insperger \& Stépán, 2004b]. This means that parametric excitation can also be introduced successfully at the time delay parameter.

In the subsequent sections, we summarize briefly the analytic results known for second-order systems, from the autonomous nondelayed system to the periodic delayed one including the delayed Mathieu equation, which requires the use of the infinite dimensional version of the Floquet Theory for time-delay systems. The sampling effect is presented as parametric excitation at the time delay, and the corresponding stability chart of the second-order system is constructed. This example serves also as a basic idea for the so-called 
semi-discretization method, which is also introduced. Investigating local bifurcations in oscillatory systems, the importance of the recognition of subcritical Hopf bifurcations is explained by simple examples. Then, as the application part of the theory and methods, the cutting process is analyzed. First, the stability and nonlinear dynamics of turning, then those of highly interrupted cutting are presented. The subcritical sense of the bifurcations has a central role in explaining the peculiar nonlinear oscillations detected in the experimental results, too. Although most of the dynamic phenomena are presented in a descriptive way, some basic calculations representing the application of the methods in a simple way are also given in detail.

\section{Parametrically Excited Delayed Oscillators}

As an introductory example for second-order and slightly damped systems subjected to delayed feedback and parametric excitation, the delayed Mathieu equation is considered in the scalar form:

$$
\ddot{x}(t)+\kappa \dot{x}(t)+(\delta+\varepsilon \cos t) x(t)=b x(t-2 \pi) .
$$

In this example, the time delay can be normalized to $2 \pi$ with an appropriate time scale transformation, equal to the time periodicity of the stiffness parameter in the same way as in the case of highly interrupted cutting models. The scalar parameter $\kappa$ is proportional to the (small) positive damping in the system, while the parameters $\delta$ and $\varepsilon$ are proportional to the mean value and the amplitude of the harmonic stiffness variation, and the parameter $b$ is proportional to the gain of the delayed feedback.

When the stiffness amplitude $\varepsilon$ and the gain $b$ are zero, the classical damped oscillator is considered in the form

$$
\varepsilon=0, b=0 \Rightarrow \ddot{x}(t)+\kappa \dot{x}(t)+\delta x(t)=0 .
$$

According to the basic theory of linear autonomous ordinary differential equations (ODEs) (proposed by Maxwell in 1865, see [Porter, 1967]), the zeros of the characteristic polynomial determine the stability properties of (2): if and only if all the characteristic roots have negative real parts, the system is asymptotically stable. The famous Routh-Hurwitz criterion (see [Routh, 1877] and [Hurwitz, 1895]) provides an algorithm to check this condition in the characteristic polynomials like

$$
\lambda^{2}+\kappa \lambda+\delta=0 .
$$

Accordingly, Eq. (2) has asymptotically stable trivial solution if and only if

$$
\kappa>0 \text { and } \delta>0 \text {. }
$$

The corresponding trivial stability is presented in Fig. 1 in order to give the first element of a complete list of charts where the stable regions are always shaded, while the blank regions refer to instability.

In the undamped and uncontrolled case, we obtain the classical Mathieu equation (see [Mathieu, 1868]):

$$
\kappa=0, b=0 \Rightarrow \ddot{x}(t)+(\delta+\varepsilon \cos t) x(t)=0 .
$$

Although, the so-called Hill's infinite determinant method was available in the literature [Hill, 1886; Rayleigh, 1887], van der Pol and Strutt [1928] published the corresponding stability chart (often referred to as Strutt-Ince chart) in analytical form only much later. The chart is shown in Fig. 2. The stability chart of the damped oscillator

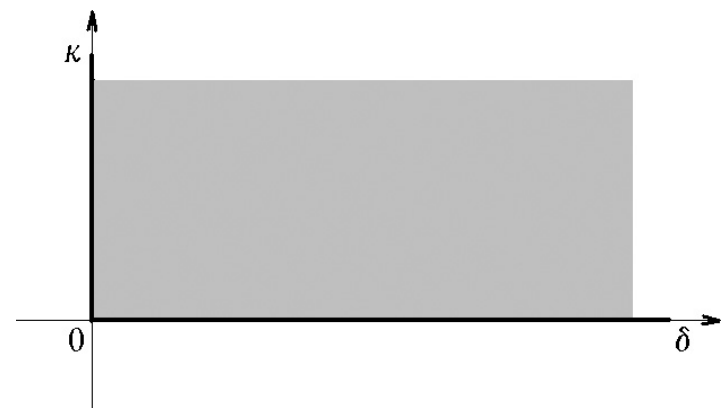

Fig. 1. Stability chart of the damped oscillator (2).

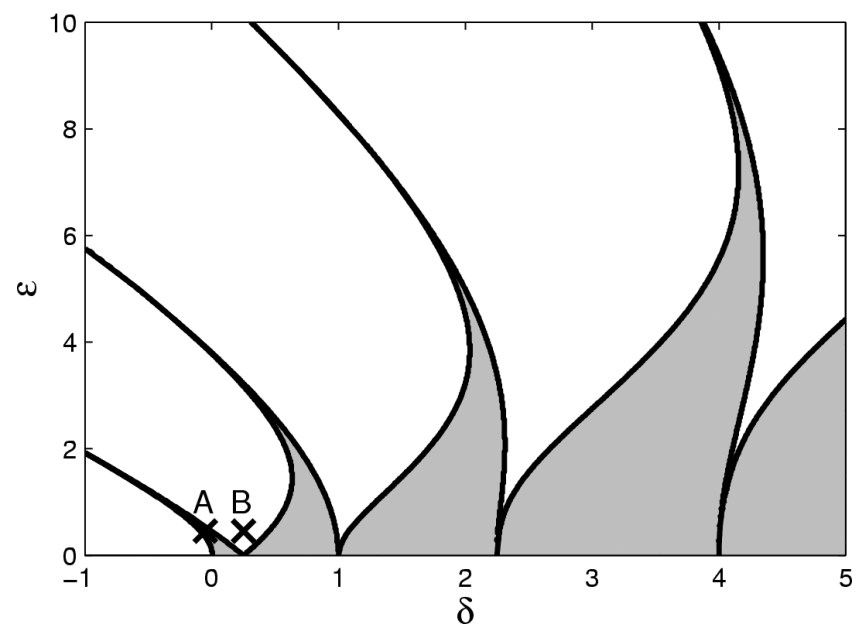

Fig. 2. Stability chart of the parametrically excited undamped oscillator, the Mathieu equation (3). 


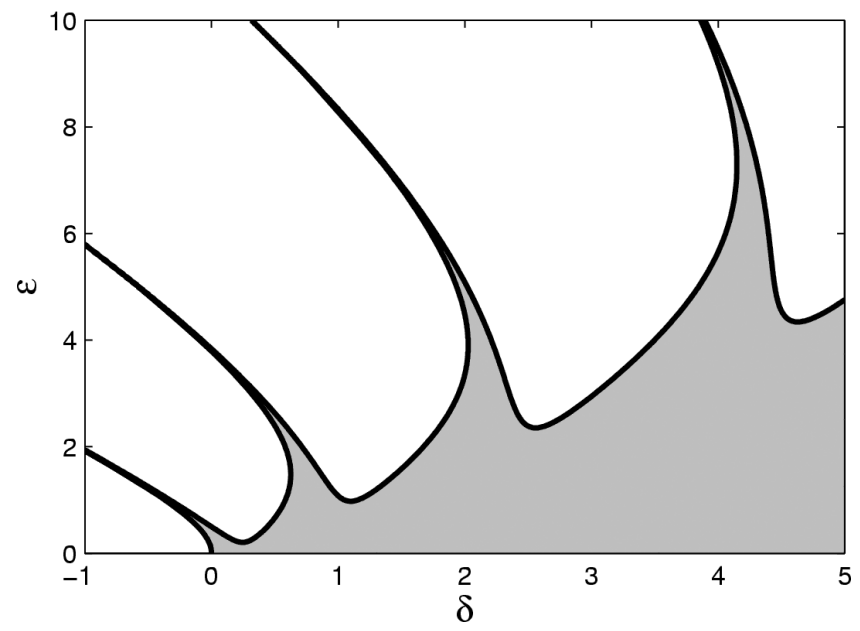

Fig. 3. Stability chart of the damped Mathieu equation with $\kappa=0.2$.

subjected to parametric excitation is given in Fig. 3. This chart is easily calculated with simple computer codes nowadays by a piecewise constant approximation of the time-periodic stiffness parameter, but it can also be constructed in the classical power series approximation way. Point A represents the stabilized inverted pendulum (see [Stevenson, 1908; Levi \& Broer, 1995]), point B represents the swing in the unstable region.

In the case of the undamped oscillator subjected to delayed feedback without parametric excitation, we have the so-called delayed oscillator equation

$$
\kappa=0, \varepsilon=0 \Rightarrow \ddot{x}(t)+\delta x(t)=b x(t-2 \pi) .
$$

With the help of the $D$-subdivision method [Neimark, 1949] applied for the characteristic function

$$
D(\lambda)=\lambda^{2}+\delta-b \mathrm{e}^{-2 \pi \lambda},
$$

it is easy to prove that the stability boundaries are straight lines in the parameter plane $(\delta, b)$. To select the stable domains among them, Bhatt and Hsu [1966] applied the method of Pontryagin [1942]. Since then, more general stability criteria have appeared in the literature, like those of Stépán [1989] or Olgac and Sipahi [2002]. The corresponding chart is presented in Fig. 4. In the presence of small damping, Fig. 5 shows the chart.

With the early paper of Halanay [1961], the infinite dimensional version of the Floquet Theory started to develop for delayed systems, but it provided only a theoretical possibility for the stability analysis of second-order systems like the

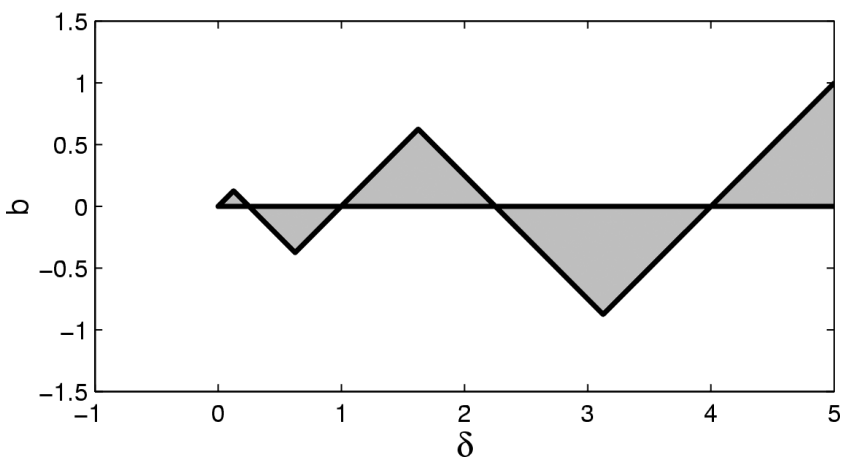

Fig. 4. Stability chart of the delayed oscillator (4).

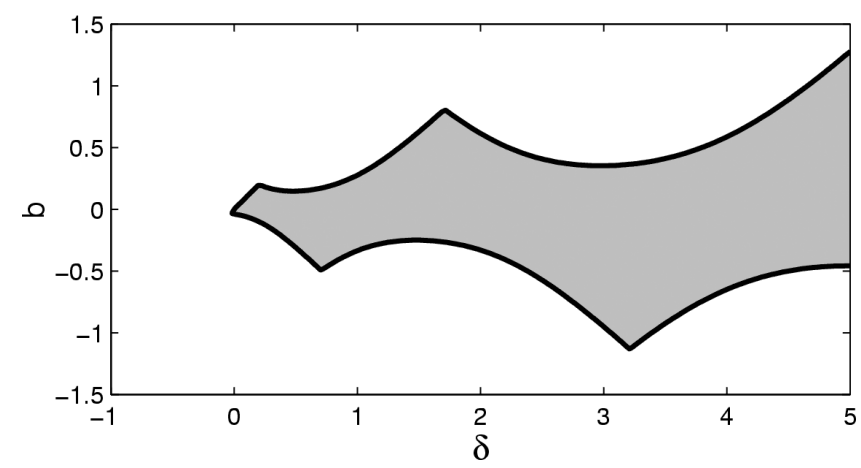

Fig. 5. Stability chart of the delayed and damped oscillator with $\kappa=0.2$.

most general case of the delayed Mathieu equation (1). After several attempts to work out an algorithm for the stability analysis of time-periodic time-delayed systems, the straight generalization of the classical Hill's infinite determinant method provided an analytic solution to the problem (see [Insperger \& Stépán, 2002a]).

In the undamped case, the delayed oscillator subjected to harmonic parametric excitation assumes the form

$$
\kappa=0 \Rightarrow \ddot{x}(t)+(\delta+\varepsilon \cos t) x(t)=b x(t-2 \pi) .
$$

It was proven, that the stability boundaries remain straight lines in the parameter plane $(\delta, b)$ for any fixed value of $\varepsilon$, and these lines are passing along the boundary curves of the Strutt-Ince diagram of Fig. 2 for varying parametric excitation amplitude $\varepsilon>0$. A typical stability chart is presented for $\varepsilon=1$ in Fig. 6 , where the triangles of the chart in Fig. 5 become somewhat smaller and separated. A three-dimensional representation of the chart is shown in Fig. 7.

With a further generalization of the above methods and results, the straight-line boundaries 


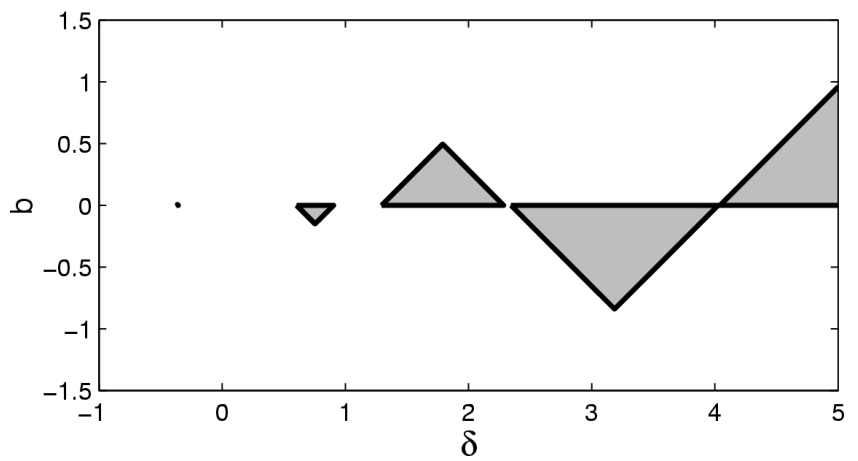

Fig. 6. Stability chart of the undamped delayed Mathieu equation (5) for $\varepsilon=1$.

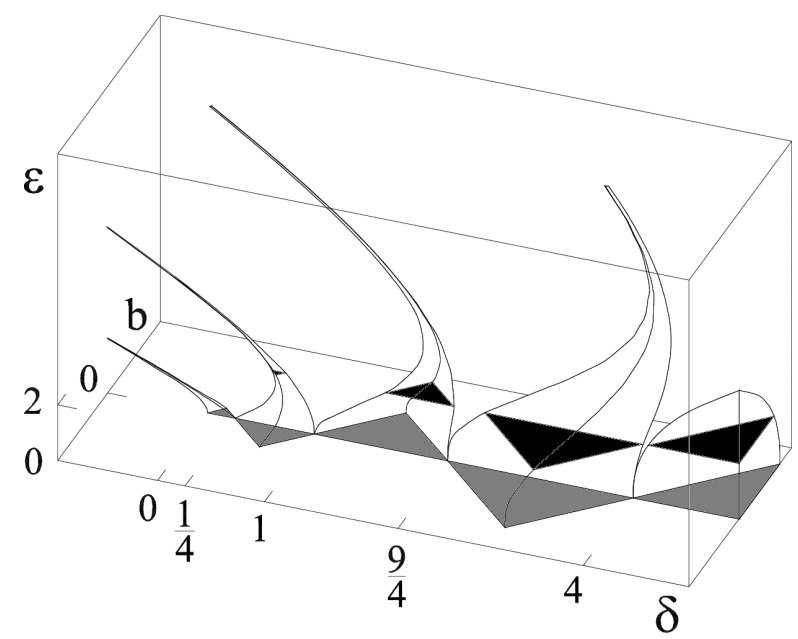

Fig. 7. Stability chart of the undamped delayed Mathieu equation (5).

were also found and proven in the most general case of the delayed Mathieu equation (1) (see [Insperger \& Stépán, 2003]). The nonzero damping merges the triangle shaped stable domains of the delayed oscillator, but the parametric excitation cuts these

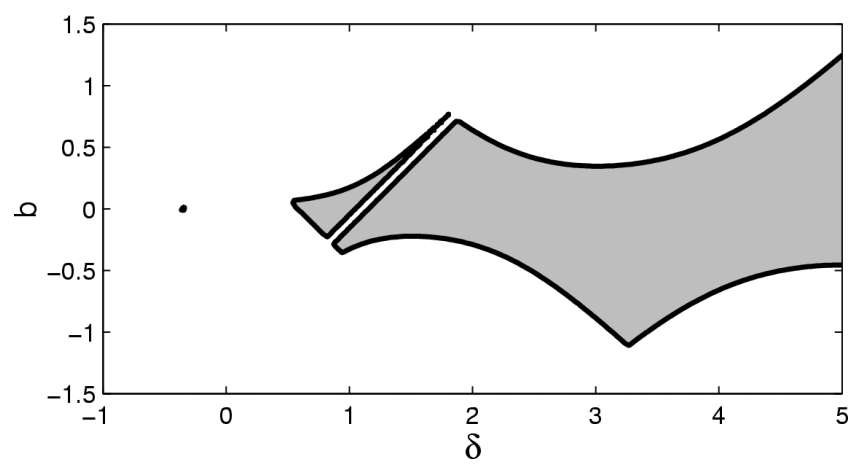

Fig. 8. Stability chart of the damped delayed Mathieu equation (1) for $\varepsilon=1$ and $\kappa=0.2$. regions by some separating lines at fixed values $\varepsilon>0$ of the excitation amplitude. A typical stability chart of Eq. (1) is presented in Fig. 8 for $\varepsilon=1$ and $\kappa=0.2$.

Rarely do the above charts directly transform to the parameter space of realistic physical problems, but they serve as unique and reliable reference examples to test numerical methods, and they also help to give an overall picture to understand the peculiar stability behavior of these time-periodic and also time-delayed second-order systems.

\section{Sampled Delayed Oscillator}

In case of the delayed Mathieu equation (1), the parametric excitation appears in the stiffness term of the system, like in case of the highly interrupted cutting model of high-speed milling (see Fig. 16). Parametric excitation can appear, however, in the time delay term, too, in the same way as explained in the Introduction for cutting with variable spindle speed. The standard model of an undamped oscillator with time-periodic feedback assumes the form

$$
\ddot{x}(t)+\delta x(t)=b x(t-\tau(t)),
$$

where $\tau$ is a periodic function of the time. Actually, the sampled and delayed feedback (including a zeroorder-holder) belongs to this class of systems. For these digital control systems, the time delay function has the form:

$$
\tau(t)=t-\Delta t\left(\operatorname{int}\left(\frac{t}{\Delta t}\right)-1\right),
$$

where $\Delta t$ denotes the sampling time, and the function is presented in Fig. 9. If $\Delta t=4 \pi / 3$, for example, then the mean time delay is just $1.5 \Delta t=2 \pi$ as in Eq. (1).

If this time delay variation is substituted in Eq. (6), then the argument assumes the form

$$
\begin{aligned}
t-\tau(t)=\Delta t\left(\operatorname{int}\left(\frac{t}{\Delta t}\right)-1\right) & =(j-1) \Delta t \\
j & =0,1,2, \ldots
\end{aligned}
$$

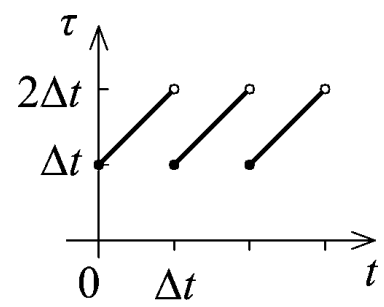

Fig. 9. Sampling effect as periodic time delay. 
and the usual form of the sampled oscillator is obtained as

$$
\begin{array}{r}
\ddot{x}(t)+\delta x(t)=b x((j-1) \Delta t), \\
t \in[j \Delta t,(j+1) \Delta t) .
\end{array}
$$

This system can be analyzed in closed form, since the right-hand side of the equation is piecewise constant, and the general solution for each sampling interval has the form

$x(t)=C_{1} \cos (\sqrt{\delta} t)+C_{2} \sin (\sqrt{\delta} t)+\frac{b}{\delta} x((j-1) \Delta t)$,

where the parameters $C_{1,2}$ have to be calculated for each sampling interval from the initial

conditions $x(j \Delta t)$ and $\dot{x}(j \Delta t)$. The lengthy algebraic calculation provides the position and velocity for this sampling interval, which serve as initial values for the next one. This way, a three-dimensional linear discrete mapping is constructed that connects the delayed position, the present position and the present velocity of the system at the sampling instants:

$$
\left(\begin{array}{c}
x(j \Delta t) \\
x((j+1) \Delta t) \\
\dot{x}((j+1) \Delta t)
\end{array}\right)=\mathbf{A}\left(\begin{array}{c}
x((j-1) \Delta t) \\
x(j \Delta t) \\
\dot{x}(j \Delta t)
\end{array}\right)
$$

with coefficient matrix

$$
\mathbf{A}=\left(\begin{array}{ccc}
0 & 1 & 0 \\
\frac{b}{\delta}(1-\cos (\sqrt{\delta} \Delta t) & \cos (\sqrt{\delta} \Delta t) & \frac{1}{\sqrt{\delta}} \sin (\sqrt{\delta} \Delta t) \\
\frac{b}{\sqrt{\delta}} \sin (\sqrt{\delta} \Delta t) & -\sqrt{\delta} \sin (\sqrt{\delta} \Delta t) & \cos (\sqrt{\delta} \Delta t)
\end{array}\right) .
$$

Clearly, the stability of the system is determined by the convergence of this vector geometric series, which depends on the eigenvalues of the matrix $\mathbf{A}$ :

$$
\begin{aligned}
\operatorname{det}(\mu \mathbf{I}-\mathbf{A})= & \mu^{3}-2 \cos (\sqrt{\delta} \Delta t) \mu^{2} \\
& +\left(1-\frac{b}{\delta}+\frac{b}{\delta} \cos (\sqrt{\delta} \Delta t)\right) \mu \\
& -\frac{b}{\delta}(1-\cos (\sqrt{\delta} \Delta t))
\end{aligned}
$$

has to be checked whether its zeros are in modulus less than 1 . The transformation $\mu=(\eta+1) /(\eta-1)$ leads to a polynomial of $\eta$ where $\left|\mu_{1,2,3}\right|<1$ is equivalent to $\operatorname{Re} \eta_{1,2,3}<0$. Thus, the Routh-

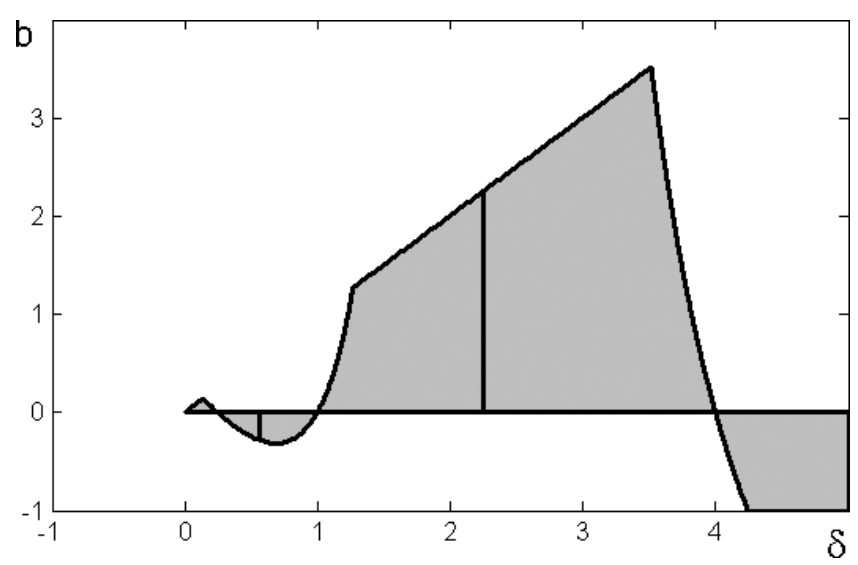

Fig. 10. Stability chart of the sampled delayed oscillator (8) for $\Delta t=4 \pi / 3$.
Hurwitz criterion can be applied for the polynomial

$$
\begin{aligned}
(1- & \left.\frac{b}{\delta}\right)\left(1-\cos (\sqrt{\delta} \Delta t) \eta^{3}+\left(1+2 \frac{b}{\delta}\right)\right. \\
\times & \left(1-\cos (\sqrt{\delta} \Delta t) \eta^{2}+(1+\cos (\sqrt{\delta} \Delta t)\right. \\
& \left.-\frac{b}{\delta}+\frac{b}{\delta} \cos (\sqrt{\delta} \Delta t)\right) \eta+(1+\cos (\sqrt{\delta} \Delta t) .
\end{aligned}
$$

The resulting necessary conditions of asymptotic stability are

$$
\begin{aligned}
& b<\delta, \quad \delta \neq \frac{k^{2} \pi^{2}}{(\Delta t)^{2}}, \quad k=1,2, \ldots \quad \text { and } \\
& 0<b<\frac{1+2 \cos (\sqrt{\delta} \Delta t)}{1-\cos (\sqrt{\delta} \Delta t)} \delta \quad \text { or } \\
& 0>b>\frac{1+2 \cos (\sqrt{\delta} \Delta t)}{1-\cos (\sqrt{\delta} \Delta t)} \delta .
\end{aligned}
$$

The stability chart in Fig. 10 is presented for the mean time delay $2 \pi$, i.e. for $\Delta t=4 \pi / 3$, so the stable domains can be compared to those of the delayed oscillator originated in analogue feedback in Fig. 4.

\section{Semi-Discretization Method}

The example of the digitally controlled oscillator as a time-periodic delay system also serves as the basic idea of a numerical method that can be applied very 

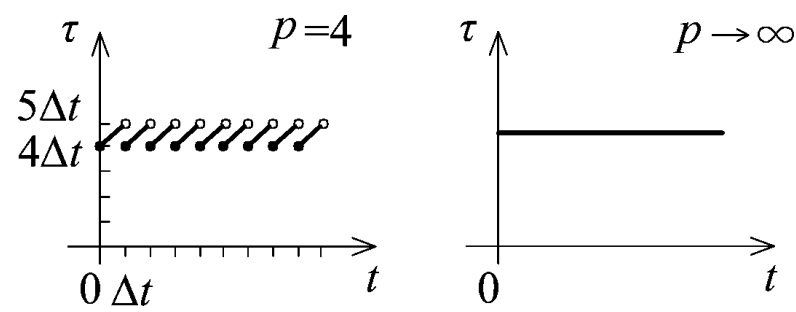

Fig. 11. Approximating constant delay by periodic ones originated in sampling.

efficiently to the stability analysis of any delayed system with parametric excitation at any (or all) of its parameters.

Consider a kind of generalization of the equation of motion (8) in the form

$$
\begin{array}{r}
\ddot{x}(t)+\delta x(t)=b x((j-p) \Delta t), \\
t \in[j \Delta t,(j+1) \Delta t)
\end{array}
$$

with any integer $p \geq 1$. Figure 11 shows the time varying time delay in the form of Eq. (9) for $p=4$. If the sampling time is chosen to be $\Delta t=2 \pi /(p+1 / 2)$ then the limit $p \rightarrow \infty$ approaches the case of the analogue control delay $2 \pi$ in (4) as shown in Fig. 11 .

Any time delay function can, however, be approximated by these piecewise sampled systems, and the corresponding further time-periodic coefficients of the systems can also be approximated by piecewise constant step functions based on the same time step $\Delta t$, too. Since the time derivatives are not discretized, the method is called semi-discretization by Insperger and Stépán [2002b]. Improved versions of the method have been developed by Insperger and Stépán [2004a] and Elbeyli and Sun [2004].

\section{Subcritical Hopf Bifurcations}

From engineering viewpoint, one of the most dangerous dynamic phenomena is the presence of an unstable limit cycle in the phase space. Engineers usually design machines and systems with linear methods. If a desired motion or stationary state is linearly stable, it may still have only a small domain of attraction, that is, it may still be sensitive for perturbations if otherwise undetectable unstable oscillations exist close to the desired states. This may already show up even in the one-degree-of-freedom model of the stick-and-slip motion of a block (see Fig. 12 with the corresponding velocity-dependent friction force $C$ ). In this case, the phase plane of the position and velocity has the well-known structure shown in Fig. 13 (see [Leine et al., 2000]).

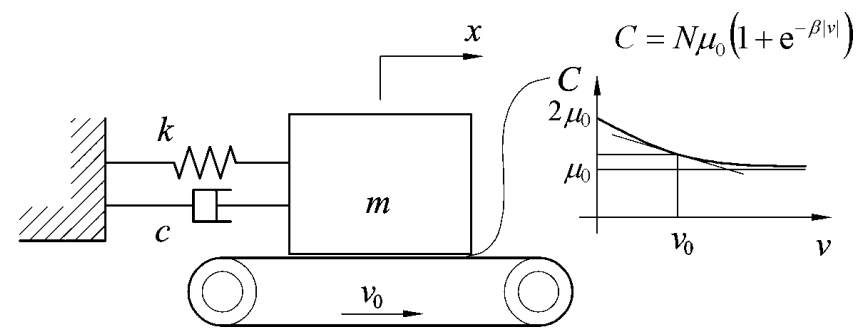

Fig. 12. Block subjected to stick-and-slip motion.

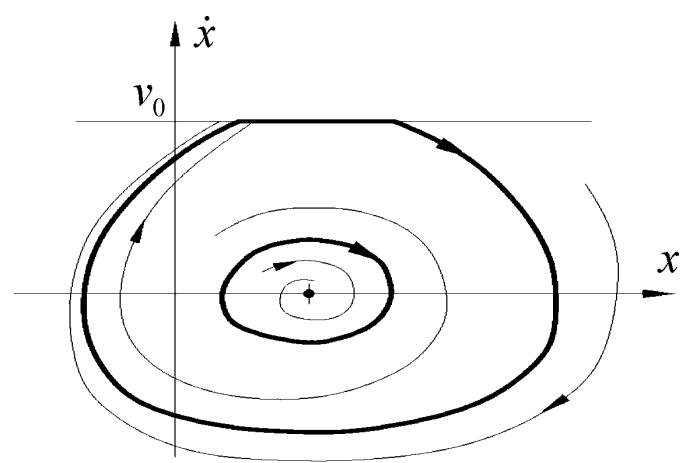

Fig. 13. Structure of phase plane for stick-and-slip motion.

The stable limit cycle around the unstable one is already a "strange attractor" in the sense that due to the non-Lipshitzian property of the friction law at zero relative velocity between the block and the belt, the solution is not unique (at least backwards in time). In this case, there is no way to have chaos (or transient chaos), since the dimension of the phase space is only 2 . In more complicated cases, the phase space dimension is 3 or more, even infinite for time-delayed and/or distributed parameter systems, and due to strong global nonlinearities, there is enough room for the trajectories to behave even chaotically outside the unstable limit cycle, also to be injected back "inside" the domain of attraction of the asymptotically stable fixed point partly bordered by the unstable limit cycle. These phenomena are present in the machine tool vibration models below.

\section{Nonlinear Modeling of Turning}

A simple mechanical model of orthogonal cutting is presented in Fig. 14. There is one cutting edge only, and it is continuously in contact with the workpiece material. The elastic tool is characterized by modal parameters like the angular natural frequency $\omega_{n}=\sqrt{k / m}$, the damping ratio $\zeta=b /\left(2 m \omega_{n}\right)$, 

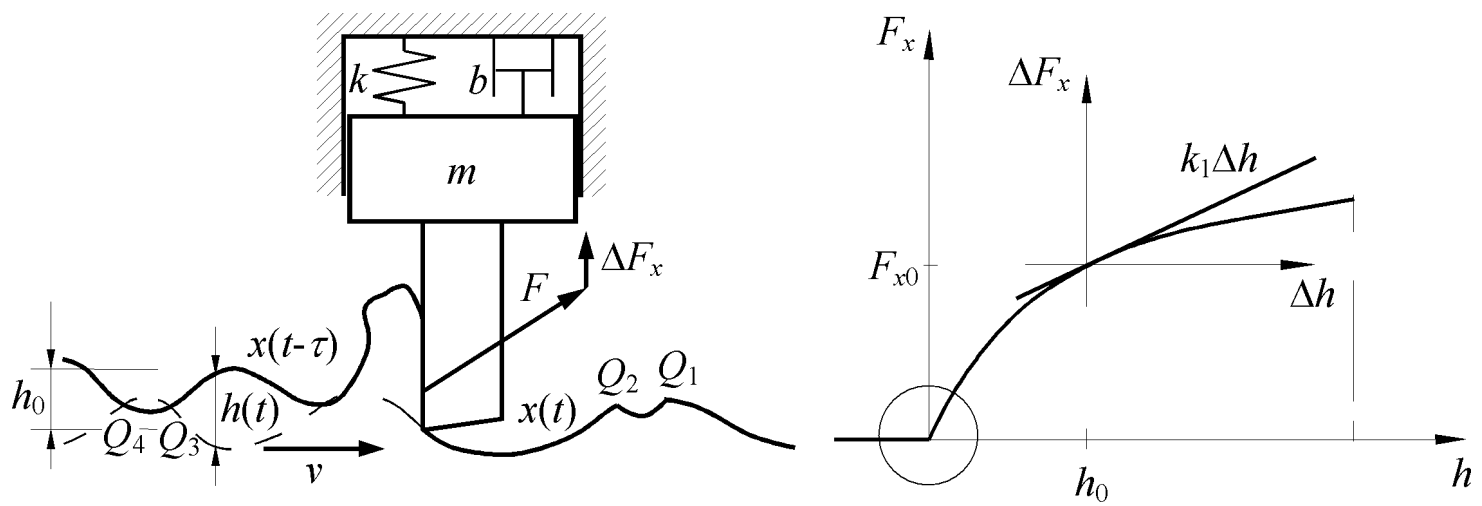

Fig. 14. Mechanical model of turning and cutting force characteristic.

and the angular frequency $\omega_{d}=\omega_{n} \sqrt{1-\zeta^{2}}$ of the damped free tool oscillation. The theoretical chip thickness is $h_{0}$, the constant chip width is $w$. The actual chip thickness is

$$
h(t)=h_{0}+x(t-\tau)-x(t) .
$$

The $x$ component of the nonlinear cutting force $F$ can be calculated in accordance with the experimentally verified three-quarter rule (see [Halley, 2000; Tlusty, 2000]):

$$
F_{x}=K w h^{3 / 4}=K w\left(h_{0}+x(t-\tau)-x(t)\right)^{3 / 4}
$$

where $K$ is an experimentally identified constant parameter. This formula is valid in a certain region around the theoretical chip thickness, but clearly, $F_{x}=0$ for negative chip thickness in the case of possible large $x$ oscillations (see the cutting force characteristics in Fig. 14). The third-degree Taylor series approximation of the cutting force variation

$$
\Delta F_{x}=F_{x}-F_{x 0}
$$

with respect to the chip thickness variation

$$
\Delta h=h(t)-h_{0}=x(t-\tau)-x(t)
$$

assumes the form

$$
\Delta F_{x} \approx k_{1}(\Delta h)-\frac{1}{8 h_{0}} k_{1}(\Delta h)^{2}+\frac{5}{96 h_{0}^{2}} k_{1}(\Delta h)^{3} .
$$

The stationary cutting force $F_{x 0}$ and the so-called cutting coefficient $k_{1}$ assume the form

$$
F_{x 0}=K w h_{0}^{3 / 4}, \quad k_{1}=\left.\frac{\partial F_{x}}{\partial h}\right|_{h_{0}}=\frac{3}{4} \frac{K w}{\sqrt[4]{h_{0}}},
$$

respectively. The equation of motion has the simple form

$$
\ddot{x}(t)+2 \zeta \omega_{n} \dot{x}(t)+\omega_{n}^{2} x(t)=\frac{1}{m} \Delta F_{x} .
$$

If the cutting force variation is substituted here using (14) and (15), and the dimensionless time $\tilde{t}=\omega_{n} t$ is introduced, then the nonlinear delaydifferential equation

$$
\begin{aligned}
x^{\prime \prime}(\tilde{t})+ & 2 \zeta x^{\prime}(\tilde{t})+(1+\tilde{w}) x(\tilde{t})-\tilde{w} x(\tilde{t}-\tilde{\tau}) \\
= & -\frac{1}{8 h_{0}} \tilde{w}(x(\tilde{t})-x(\tilde{t}-\tilde{\tau}))^{2} \\
& +\frac{5}{96 h_{0}^{2}} \tilde{w}(x(\tilde{t})-x(\tilde{t}-\tilde{\tau}))^{3}
\end{aligned}
$$

is obtained with its linear part on the left-hand side. The dimensionless chip width $\tilde{w}$ can be calculated as the ratio of the cutting coefficient and the modal stiffness of the machine tool:

$$
\tilde{w}=\frac{k_{1}}{m \omega_{n}^{2}}=\frac{k_{1}}{k}=\frac{3}{4} \frac{K}{k \sqrt[4]{h_{0}}} w,
$$

while the dimensionless time delay $\tilde{\tau}=\omega_{n} \tau$ and the dimensionless angular velocity $\tilde{\Omega}=\Omega / \omega_{n}$ are related to the angular velocity $\Omega$ of the cylindrical workpiece in the following way:

$$
\tilde{\tau}=\frac{2 \pi}{\tilde{\Omega}}=\frac{2 \pi}{\Omega} \omega_{n}=\tau \omega_{n} .
$$

The stability analysis of the stationary turning means the investigation of the trivial solution of the delay-differential equation (17) that can be based on the characteristic function similar to that of Eq. (4):

$$
\lambda^{2}+2 \zeta \lambda+(1+\tilde{w})-\tilde{w} \exp \left(\frac{-2 \pi \lambda}{\tilde{\Omega}}\right)=0 .
$$

The locations of the infinitely many characteristic roots $\lambda$ depend on three dimensionless parameters only. The so-called stability chart is presented in the plane of the parameters $\tilde{\Omega}$ and $\tilde{w}$ related to the chosen technology, while the modal damping $\zeta$ of 


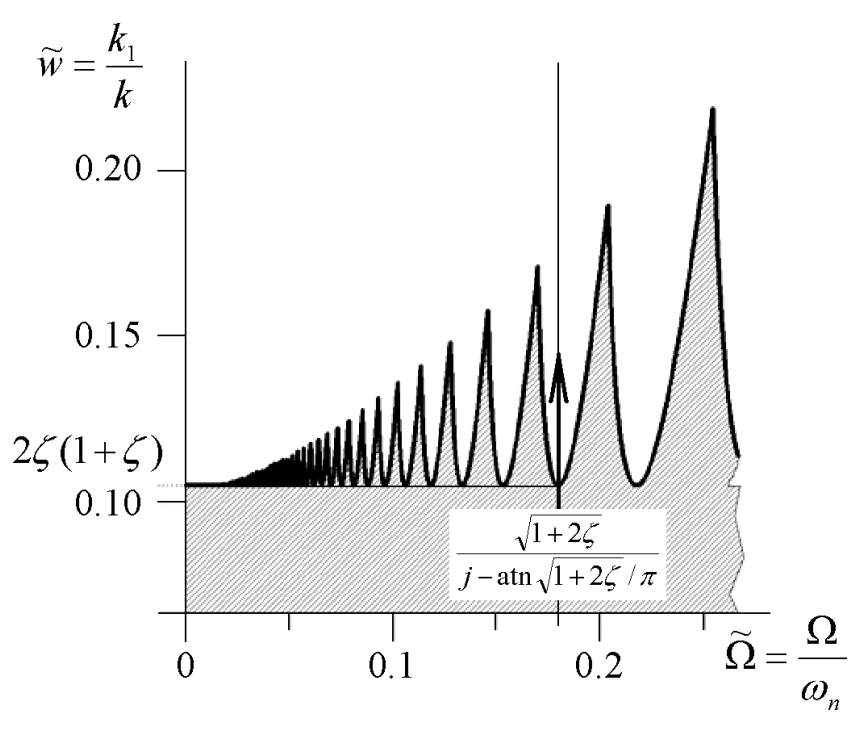

Fig. 15. Stability chart of turning.

the machine tool is a fixed parameter chosen to be 0.05 here. The shaded region in the chart of Fig. 15 shows the parameter domain where the characteristic roots are in the left-half of the complex plane, that is, where the stationary cutting is stable. This chart is a kind of transformation of the one in Fig. 5.

We briefly summarize the results of the Hopf bifurcation analysis at the minimum points of the stability lobes (also pointed out in Fig. 15 at the $j$ th lobe) to make them comparable to the special nonlinear phenomena of high-speed milling. The lengthy infinite dimensional center manifold reduction and the normal form calculation (see [Stépán \& Kalmár-Nagy, 1997; Kalmár-Nagy et al., 2001; Gilsinn, 2002]) proves the subcritical sense of the bifurcation, that is, unstable periodic vibration exists around the stable stationary cutting approximated in the form

$$
\begin{aligned}
x(t)= & \frac{8}{\sqrt{5}} h_{0}\left(1+\frac{11}{30} \zeta\right) \sqrt{1-\frac{\tilde{w}}{2 \zeta(1+\zeta)}} \\
& \times \cos \left(\sqrt{1+2 \zeta} \omega_{n} t\right) .
\end{aligned}
$$

Since there are unstable periodic motions, the existence of a global attractor is not explained by the local bifurcation analysis presented in the previous section. Experiments (see [Stépán, 2001b]) and numerical simulations show that there is no attractor in the delayed system - the large amplitude stable motion exists only when the tool leaves the workpiece for certain time periods. As Fig. 14 shows, the vibration amplitudes of the tool may become so large that the tool is not in contact with the workpiece between points $Q_{1}$ and $Q_{2}$, then between $Q_{3}$ and $Q_{4}$, and so on. In these intervals, there is zero cutting force, no regenerative effect, and the system is described by the equation of the damped oscillator:

$$
x^{\prime \prime}(\tilde{t})+2 \zeta x^{\prime}(\tilde{t})+(1+\tilde{w}) x(\tilde{t})=0 .
$$

Since the trivial solution in this damped oscillator is exponentially stable (just like Eq. (2)), the tool quickly enters the material of the workpiece again, and the destabilizing delay effect is switched on again. The system will have switches between these two dynamics: the damped free-flight of the tool described by (22) and the regenerative cutting described by (17). This may be periodic, quasi-periodic, chaotic or transient chaotic motion [Stépán, 2001b]. The geometric structure of the corresponding infinite dimensional phase space is presented in [Stépán et al., 2004]. This structure shows complicated attractors outside the unstable limit cycles - somewhat similar to the case of the simple stick-and-slip dynamics explained above.

The above-described phase space structure represents a cutting process where the free-flights of the tool are regulated by the cutting process itself. This is called self-interrupted cutting. Milling has a qualitatively different global dynamics in this respect.

\section{Nonlinear Modeling of Milling}

High-speed milling is one of the most preferred and efficient cutting processes nowadays. High-speed milling has specific properties like small tool diameter, low number of milling teeth (typically, only $z=$ 2 ), and high cutting speed. This means that, most of the time, none of the tool cutting edges is in contact with the workpiece, while cutting occurs during those short time-intervals only when one of the teeth hits the workpiece. In this respect, the model includes the classical regenerative effect in the same way as described for turning. There is one major difference, though — the periods of no-contact are regulated by the cutting speed parameter, and not by an unstable cutting process dynamics itself. Accordingly, high-speed milling is a kind of parametrically interrupted cutting as opposed to the self-interrupted cutting arising in unstable turning processes. All this means, that the corresponding mathematical model is a second-order scalar delaydifferential equation with time-periodic coefficients in it (see [Insperger et al., 2003a, 2003b; Insperger \& Stépán, 2004c]), just like the delayed Mathieu equation (1). To analyze the dynamics of the milling 
process, it is essential to understand the stability properties of (1) given in the first part of this paper. In what follows, we discuss a special case, when the analysis can be somewhat simplified.

Actually, the time spent cutting to not cutting may be less than $10 \%$, so it can often be considered as a small parameter. This case is called highly interrupted cutting. For highly interrupted cutting, the short contact periods between the tool and the workpiece are often modeled as kind of impacts, where the linear impulse coming from the cutting force contains a past-effect, i.e. the same regenerative effect as in the case of turning. The corresponding mathematical model is similar to that of an impact oscillator that also involves time delay. In other words, it is a delay-differential equation with time-periodic coefficients, where the timeperiodicity is far from harmonic, rather close to periodic Dirac-delta impulses. In this case, the governing equations of the tool free-flights and the subsequent impacts can be solved analytically, and a closed form nonlinear Poincaré mapping can be constructed for this parametrically excited timedelayed system.

Figure 16 presents a simple one-degree-offreedom model of highly interrupted cutting. Here, the number of cutting edges is virtually one, and it is in contact with the workpiece material periodically with time period $\tau$. The time spent in contact is $\rho \tau$ where $\rho \ll 1$ is the ratio of time spent cutting to time period. Clearly, the time delay $\tau$ is the time period of the tool revolution over the number $z$ of cutting edges for cases of more edges than one. If $\Omega$ denotes the tool angular velocity then the time period, and also the delay, is $\tau=2 \pi /(z \Omega)$.

The elastic tool is characterized with the same modal parameters as in case of turning, the chip geometry and the cutting force characteristic is also

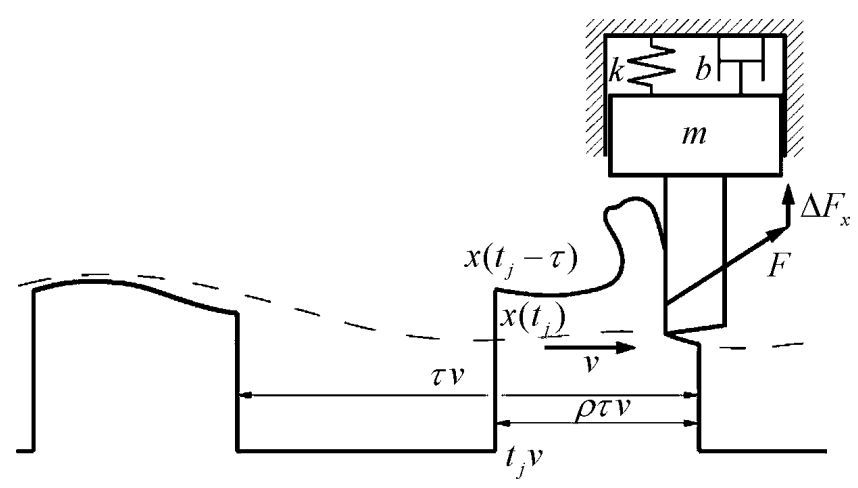

Fig. 16. Mechanical model of highly interrupted cutting. the same during the contact periods (see Fig. 14). Consequently, the actual chip thickness is given either by Eq. (10) for the contact periods, or it is zero for no contact. The cutting force and its variation, also its third degree power series, can be given by the same formulae as in (11)-(15) for turning, again. The contact time $\rho \tau$ is considered to be so short that the position of the tool, and also the chip thickness, do not change during this time. Davies et al. [2000, 2002], and Bayly et al. [2001] analyzed this approximation theoretically and experimentally, too. The equations of motion can be constructed for the two parts of the tool motion in the following way.

For the free flight of the tool, we have

$$
\begin{array}{r}
\ddot{x}(t)+2 \zeta \omega_{n} \dot{x}(t)+\omega_{n}^{2} x(t)=0, \\
t \in\left[t_{j}-\tau, t_{j}-\rho \tau\right),
\end{array}
$$

where $t_{j}-\rho \tau$ is the initial time instant of the $j$ th contact period between the tool and the workpiece $(j=1,2, \ldots)$. The corresponding initial conditions are

$$
x_{j-1}=x\left(t_{j-1}\right) \approx x\left(t_{j-1}-\rho \tau\right), \quad v_{j-1}=\dot{x}\left(t_{j-1}\right),
$$

where, again, we consider that the position of the tool does not change much during the short contact period.

For the contact period, the usual condition of the classical impact theory is applied: all the forces except the contact ones (actually, except the cutting force) are negligible. Using also formula (11) of the cutting force, we have

$$
\begin{aligned}
m \ddot{x}(t) & \approx F_{x}(x(t), x(t-\tau)) \\
& \approx K w\left(h_{0}+x_{j-1}-x_{j}\right)^{3 / 4}, \quad t \in\left[t_{j}-\rho \tau, t_{j}\right),
\end{aligned}
$$

where the initial conditions are as follows

$$
x_{j}=x\left(t_{j}-\rho \tau\right) \approx x\left(t_{j}\right), \quad v_{j}^{-}=\dot{x}\left(t_{j}-\rho \tau\right) .
$$

The Taylor series of this cutting force with respect to the chip thickness deviation $x_{j-1}-x_{j}$ of the $j$ th contact period assumes the same form as (14):

$$
\begin{aligned}
F_{x} \approx & F_{0 x}+k_{1}\left(x_{j-1}-x_{j}\right)-\frac{1}{8 h_{0}} k_{1}\left(x_{j-1}-x_{j}\right)^{2} \\
& +\frac{5}{96 h_{0}^{2}} k_{1}\left(x_{j-1}-x_{j}\right)^{3} .
\end{aligned}
$$

First, we briefly summarize the linear stability results and the corresponding nonlinear vibrations derived with the above "small" $\rho$ condition. 
The closed-form solutions of the linear ordinary differential equations (23) and (25) lead to a nonlinear discrete mapping of the form

$$
\begin{aligned}
& {\left[\begin{array}{l}
x_{j} \\
v_{j}
\end{array}\right]=\mathbf{A}\left[\begin{array}{l}
x_{j-1} \\
v_{j-1}
\end{array}\right]+\left[\sum_{h+k=2,3 ; h, k \geq 0} b_{h k} x_{j-1}^{h} v_{j-1}^{k}\right]} \\
& +\left[\begin{array}{c}
0 \\
\frac{\rho \tau}{m} F_{0 x}
\end{array}\right] \text {. }
\end{aligned}
$$

The detailed expressions of the coefficient matrix $\mathbf{A}$ and the nonlinear coefficients $b_{h k}$ are given in [Szalai, 2002; Szalai \& Stépán, 2003]. The lengthy algebraic analysis of the linear part shows the possibility of both Neimark-Sacker (or secondary Hopf) and period doubling (or flip) bifurcations (see [Guckenheimer \& Holmes, 1986]). The corresponding stability chart in the plane of $z \tilde{\Omega}$ (the product of the number of cutting edges and dimensionless cutting speed), and $\rho \tilde{w}$ (the product of the ratio of time spent cutting to not cutting and the dimensionless chip width) is presented in Fig. 17 for damping ratio $\zeta=0.02$. The number of lobes is doubled compared to that of the chart for turning.

The corresponding bifurcation diagram of Fig. 18 shows the arising vibration amplitudes for stable and unstable stationary cutting and period2 oscillations at the dimensionless cutting speed denoted by a thick arrow $(z \tilde{\Omega}=2.4)$ in the stability chart at a flip lobe.

The bifurcation diagram is constructed from simulation results, but it fits perfectly to the results of the algebraic predictions: the flip bifurcation at $\tilde{w}_{c r}$ is subcritical, and unstable period-2 oscillations exist around the stable stationary cutting. This local bifurcation is similar to that of turning, and it is also related to the local asymmetric nonlinearity of the cutting force around the theoretical chip thickness.

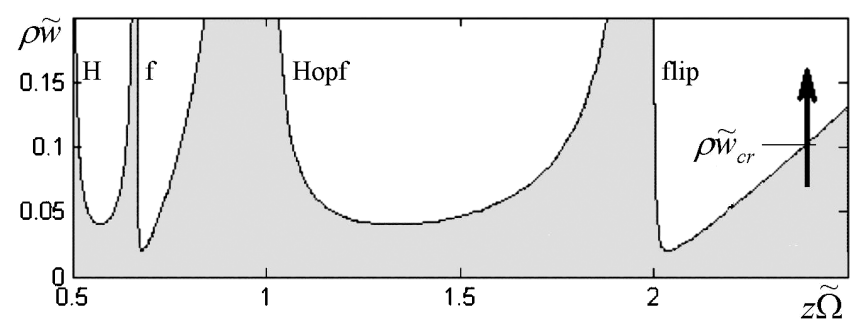

Fig. 17. Stability chart of milling in case of highly interrupted cutting.

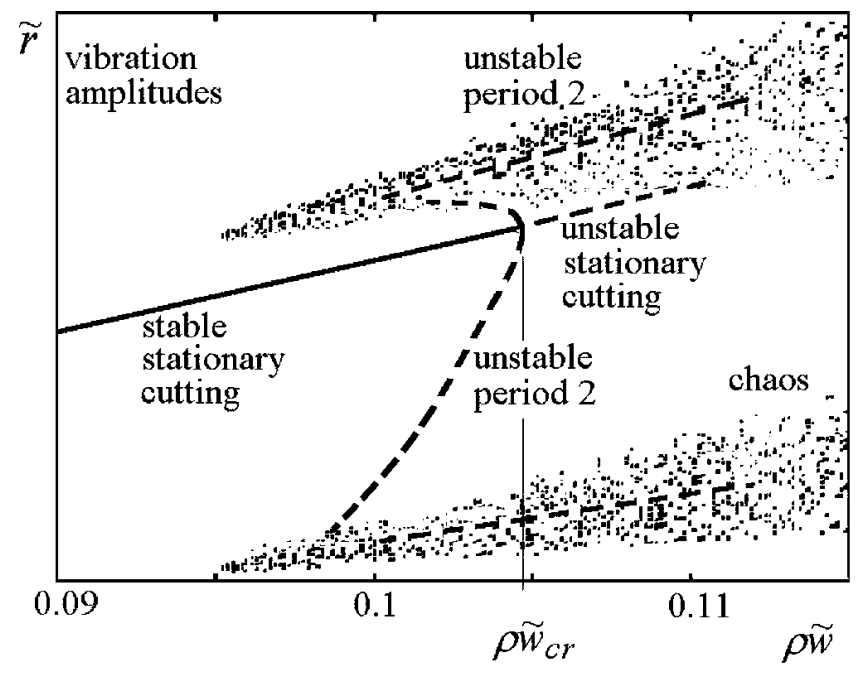

Fig. 18. Bifurcation diagram at a flip lobe of highly interrupted cutting.

It can also be shown analytically, that there exists a large amplitude stable period-2 oscillation outside the unstable branches. The existence of this kind of motion is related to the so-called fly-over effect, when the tool misses the contact with the workpiece at every second period (see the schematic picture of a period-2 oscillation with fly-over in Fig. 16). In the case of a two-flute end mill, this oscillation means that only one of the cutting edges hits the workpiece, the other one always misses it. This nonlinear oscillation is related to the another nonlinearity of the cutting force characteristic encircled at zero chip thickness in Fig. 14. This period-2 fly-over can be stable, but it can also become unstable, and can bifurcate into chaos through further period-doubling bifurcations. The bifurcation diagram in Fig. 18 shows this chaotic case, when both period-2 oscillations are unstable. The existence of chaotic oscillations is actually proven in the corresponding nonlinear discrete model (18) (see [Szalai et al., 2004]).

Mann et al. [2003] have constructed an experimental stability chart for highly interrupted cutting. Aluminium test samples were centrally milled by means of carbid end mill with a single flute (the second flute was ground off). The ratio $\rho$ of the time spent cutting to the tooth pass period $\tau$ was $\rho=$ 0.108 . The experimental observations confirmed the linear theory well; they followed the theoretically predicted two kinds of chatter regions. The Fourier spectrum of the time signals helped to identify the quasi-periodic and the period- 2 oscillations in the unstable cases, since the corresponding frequency 
components were also predicted analytically above the stability chart following [Insperger et al., 2003a].

Since the bifurcations along the stability limits are subcritical, it is an interesting question, what kinds of vibrations are measured in those cases when the stationary milling process is unstable. In the case of the flip lobe boundary, for example, stable period-2 oscillation may be detected with the so-called fly-over effect (see also Fig. 16). Although, the spectral analysis of the signals can provide enough evidence on the correctness of the linear predictions, further signal analysis should also prove the above described fly-over effect in the case of stable period-2 oscillations. The signals, however, were too noisy to reconstruct the phase space trajectories from the measured signals.

The application of the method of Gradišek et al. [2002] can help to extract the deterministic component of noisy signals. Using the measurement data sampled stroboscopically by $t_{s}$, and using the classical technique of presenting the trajectory in the space of $8 t_{s}$-delayed coordinates, the path of the tool can be reconstructed. One can do this reconstruction without high-speed camera shots of the cutting process that has high-speed dynamics, anyway.

The reconstructed deterministic trajectories showed the structure of the corresponding vibrations very clearly. There were stable period-2 oscillations, but their structure was not as predicted in the theory: the tool hits the workpiece at each tooth-pass period, and the predicted fly-over effect at every second tooth-pass did not show up in the experiments.

One possible explanation for this contradiction is based on the weakness of the condition on impactlike cutting, that is the parameter $\rho$ that describes the ratio of time spent cutting to tooth-pass period, cannot be considered as a small parameter even if it is in the range of 0.1 only. The so-called collocation method of Engelborghs et al. [2000] gives the possibility to calculate the global stable period-2 oscillations even when the impact condition is dropped in the Eq. (25) of motion. The spring and damping terms are not negligible compared to the cutting force $F_{x}$, the tool may leave the workpiece material during the short possible contact period $\rho \tau$, so it is not the sudden velocity variation that is modeled, but also the variation of the displacement. On one hand, this means, that the parametrically excited delayed oscillator cannot be approximated in the closed form of the discrete map (28), on the other hand, the actual contact time can be shorter than $\rho \tau$. Since the collocation method applied for the nonhighly interrupted cutting model gave results that qualitatively agreed with the enhanced experimental results, the deep analyses of the general case was unavoidable.

If the ratio $\rho$ of time spent cutting to tooth-pass period cannot be considered as a small parameter, the impact-like modeling of the tool-workpiece contact in Eq. (25) should be modified, and condition (24) on the nonvariation of the tool position is not valid anymore. Actually, the two piecewise linear equations (23) and (25) of motion can be rewritten more generally as

$$
\begin{array}{r}
\ddot{x}(t)+2 \zeta \omega_{n} \dot{x}(t)+\omega_{n}^{2} x(t) \\
=g(t) \frac{1}{m} F_{x}(x(t), x(t-\tau)), \\
g(t)=\left\{\begin{array}{ll}
0 & \text { if } t \in\left[t_{j}-\tau, t_{j}-\rho \tau\right) \\
1 & \text { if } t \in\left[t_{j}-\rho \tau, t_{j}\right)
\end{array},\right.
\end{array}
$$

where the cutting force $F_{x}$ can be formulated as in Eq. (11) in accordance with Fig. 14. The linear part of (29) at zero is already similar to the delayed Mathieu equation (1). These equations can efficiently be analyzed by the semi-discretization method generalized in Sec. 4 from the example of the sampled oscillator.

The linear stability analysis of (29) serves some surprising results as shown by Szalai and Stépán [2003]. While the instability lobes in the stability chart of the dimensionless cutting speed and chip width seem to have a double lobe system for vanishing $\rho$ parameters (see Fig. 17), the lobes related to period-doubling become closed curves for finite $\rho$ values. The stability charts are formed from a system of lobes and lenses, with lobes referring to secondary Hopf bifurcations (quasi-periodic oscillations), and lenses referring to flip bifurcations (period-2 oscillations).

An example of stability chart is presented in Fig. 19 for the same damping ratio $\zeta=0.02$ as in Fig. 17, but for a nonvanishing $\rho=0.1$. The parameter range is presented for lower cutting speeds where the difference between the lobe and lens system is more spectacular, while the dimensionless chip width is defined without the $\rho$ parameter, which explains why these numerical values are one range larger in Fig. 19 than in Fig. 17.

The difference between the lobe and lens system is less dramatic for the case of the experimental parameters $\zeta=0.0038$ and $\rho=0.108$ used by Mann 


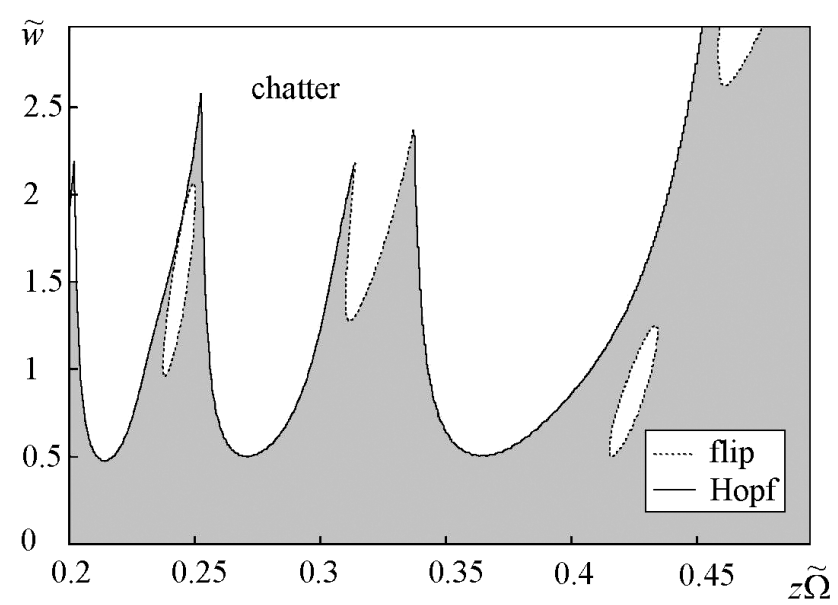

Fig. 19. Stability chart of milling (nonhighly interrupted cutting).

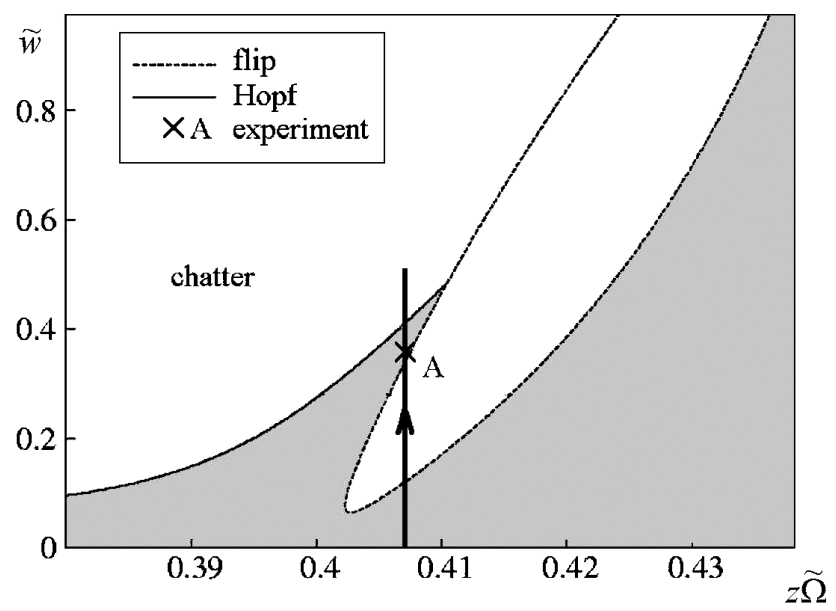

Fig. 20. Stability chart for high-speed milling experiment.

et al. [2003]. The corresponding stability chart is presented in Fig. 20, and the experimental results fit to this linear theory well (see [Stépán et al. 2004]).

An interesting experimental parameter setting is denoted at the measurement point $\mathrm{A}$ in the narrow stable band between the (secondary) Hopf lobe and the flip lens. At the parameter point A of the stability chart in Fig. 20, the experiments showed very different dynamic behaviors depending on the external noise and perturbations on the milling process. Sometimes, the system exhibited stable cutting, sometimes, however, the milling process changed to (jumped into) a complicated nonlinear oscillation. This means that the system has stable stationary cutting and also complex stable nonlinear oscillation originated in a kind of bifurcated global period-2 oscillation, together. The highly interrupted cutting model cannot explain this phenomenon since the flip stability boundaries are lobes and not lenses there.

In order to understand this nonlinear behavior of high-speed milling, a bifurcation diagram was calculated along the thick arrow in Fig. 20 at the fixed dimensionless cutting speed $z \tilde{\Omega}=0.407(3450 \mathrm{rpm})$ used also at the parameter point $\mathrm{A}$. The diagram in Fig. 21 is constructed by the use of path-following methods, and shows a much more sophisticated and partly unexplored structure than that of the diagram in Fig. 18. Note that the dimensionless amplitude $\hat{r}$ in Fig. 21 is the variation of the amplitude $\tilde{r}$ around the stationary vibration amplitude $\tilde{r}_{\text {stat }}$, i.e. $\hat{r}=\tilde{r}-\tilde{r}_{\text {stat }}$.

The flip bifurcations at the two opposite sides of the flip lens at $\tilde{w}_{1}$ and $\tilde{w}_{2}$ are subcritical and supercritical, respectively. Between these two chip thickness values, the stationary milling process is unstable, while unstable and stable period-2 oscillations arise below these critical values, respectively. The unstable period- 2 oscillation turns to a stable one at a fold bifurcation. Still, the secondary Hopf bifurcation of this global stable period-2 motion leads soon to an even more complicated stable quasi-periodic motion here, which was not possible to follow by the applied numerical method, and so it is not presented in the diagram.

The other branch, the stable period- 2 motion born in the supercritical flip bifurcation at the top of the lens, goes through a window of secondary Hopf bifurcations very quickly, then the fold shows up, and the global period-2 motion changes to unstable. It is still unclear, how many, and what kinds of stable motions exist above the flip lens and below the Hopf lobe, but as experiments show: this region might be favorable for high-efficiency stable stationary cutting, but it is very sensitive for small perturbations due to the presence of several stable and unstable nonlinear oscillations there.

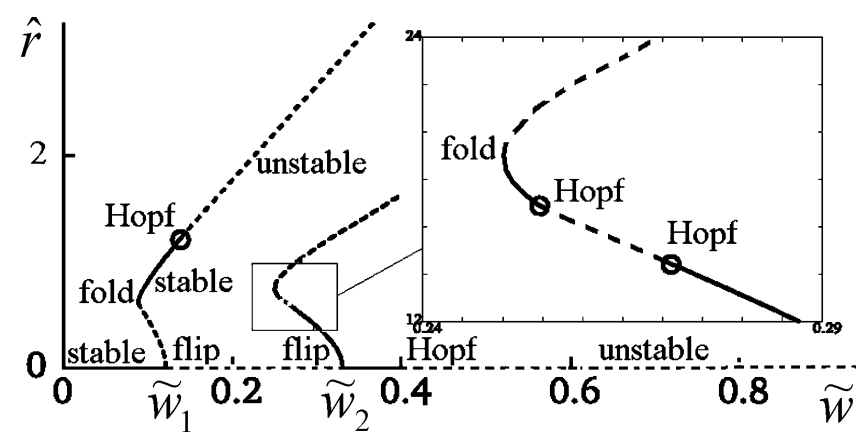

Fig. 21. Bifurcation diagram for high-speed milling through parameter point A. 


\section{Conclusions}

The stability chart of the delayed Mathieu equation is a unique analytical result for parametrically excited delayed oscillators. This chart serves as a reliable reference example to test numerical methods developed for these time-periodic and also time-delayed second-order systems. One of the numerical tools is the so-called semi-discretization method originated in the analytical study of the sampled oscillator. These mathematical tools, together with the methods of classical bifurcation theory, collocation method, and further variations of path-following methods make it possible to have an access for the surprisingly unpredictable dynamics of delayed and parametrically excited oscillators.

As one of the hardest problems in this category, the stability charts of milling processes were compared for high-speed milling with and without the use of the condition on highly interrupted cutting. The results were compared to experiments. The stability charts consisting of a double lobe system for the highly interrupted cutting case may look qualitatively surprisingly different form the lobe and lens system of the more precise models, but the quantitative difference did not seem to be important from linear stability viewpoint in the parameter range of the experiments.

Considering the nonlinear dynamics of the high-speed milling process, however, we find essential differences between the two models. Actually, the highly interrupted cutting model is unable to explain some of the peculiar multistable behavior experienced at certain parameter points. The most complicated system of stable and unstable oscillations is detected at those regions between the lobes and lenses where the efficiency of high-speed milling would be just the best. The bifurcation diagrams provide an initial guide for understanding some of these dynamic behaviors.

\section{Acknowledgments}

The authors greatly acknowledge the financial support of this work provided by the Hungarian National Science Foundation under grant nos. T043368 and F047318, the Hungarian Academy of Sciences, Research Group on Dynamics of Machines and Vehicles and Zoltán Magyary Postdoctoral Fellowship of Foundation for Hungarian Higher Education and Research.

\section{References}

Altintas, Y. \& Budak, E. [1995] "Analytical prediction of stability lobes in milling," Ann. CIRP 44, 357362.

Balachandran, B. [2001] "Non-linear dynamics of milling process," Philos. Trans. Roy. Soc. 359, 793820.

Bayly, P. V., Halley, J. E., Mann, B. P. \& Davies, M. A. [2001] "Stability of interrupted cutting by temporal finite element analysis," in Proc. ASME 2001 Design Engineering Technical Conf., Pittsburgh, paper no. DETC2001/VIB-21581 (CD-ROM).

Bellman, R. \& Cooke, K. [1963] Differential-Difference Equations (Academic Press, NY).

Bhatt, S. J. \& Hsu, C. S. [1966] "Stability criteria for second-order dynamical systems with time lag," ASME J. Appl. Mech. 33, 113-118.

Campbell, S. A. [1999] "Stability and bifurcation of a simple neural network with multiple time delays," Fields Instit. Commun. 21, 65-79.

Corpus, W. T. \& Endres, W. J. [2004] "Added stability lobes in machining processes that exhibit periodic time variation - Part 1: An analytical solution," J. Manuf. Sci. Engin. 126, 467-474.

Craig, J. J. [1986] Introduction to Robot Mechanics and Control (Addison-Wesley, Reading MA).

Davies, M. A., Pratt, J. R., Dutterer, B. \& Burns, T. J. [2000] "The stability of low immersion milling," Ann. CIRP 49, 37-40.

Davies, M. A., Pratt, J. R., Dutterer, B. S. \& Burns, T. J. [2002] "Stability prediction for low radial immersion milling," J. Manuf. Sci. Engin. 124, 217-225.

Diekmann, O., van Gils, S. A., Lunel, S. M. V. \& Walther, H.-O. [1995] Delay Equations (Springer, NY).

Elbeyli, O. \& Sun, J. Q. [2004] "On the semidiscretization method for feedback control design of linear systems with time delay," J. Sound Vibr. 273, 429-440.

Elbeyli, O., Sun, J. Q. \& Ünal, G. [2005] "A semidiscretization method for delayed stochastic systems," Commun. Nonlin. Sci. Numer. Simul. 10, 85-94.

Engelborghs, K., Luzyanina, T., Hout, K. J. \& Roose, D. [2000] "Collocation method for the computation of periodic solutions of delay differential equations," SIAM J. Sci. Compu. 22, 1953-1609.

Faassen, R. P. H., van de Wouw, N., Oosterling, J. A. J. \& Nijmeijer, H. [2003] "Prediction of regenerative chatter by modeling and analysis of high-speed milling," Int. J. Mach. Tools Manuf. 43, 14371446.

Floquet, M. G. [1883] "Equations différentielles linéaires a coefficients péridiques'," Ann. Scientifiques de l'Ecole Normale Supérieure 12, 47-89.

Gilsinn, D. E. [2002] "Estimating critical Hopf bifurcation parameters for a second order delay-differential 
equation with application to machine tool chatter," Nonlin. Dyn. 30, 103-154.

Gouskov, A. M., Voronov, S. A., Paris, H. \& Batzer, S. A. [2002] "Nonlinear dynamics of a machining system with two interdependent delays," Commun. Nonlin. Sci. Numer. Simul. 7, 207-221.

Gradišek, J., Friedrich, R., Govekar, E. \& Grabec, I. [2002] "Analysis of data from periodically forced stochastic processes," Phys. Lett. A294(3-4), 234238.

Guckenheimer, J. \& Holmes, P. [1986] Nonlinear Oscillation, Dynamical Systems and Bifurcation of Vector Fields (Springer-Verlag, NY).

Halanay, A. [1961] "Stability theory of linear periodic systems with delay (in Russian)," Revue de Mathématiques Pures et Appliquées 6, 633-653.

Halanay, A. [1966] Differential Equations: Stability, Oscillations, Time Lags (Academic Press, NY).

Hale, J. K. \& Lunel, S. M. V. [1993] Introduction to Functional Differential Equations (Springer-Verlag, NY).

Haller, G. [2004] "Exact theory of unsteady separation for two-dimensional flows," J. Fluid Mech., in press.

Halley, J. E. [2000] "Stability of low radial immersion milling," MSc thesis, Washington University, St. Louis, Missouri.

Hill, G. W. [1886] "On the part of the motion of the lunar perigee which is a function of the mean motions of the sun and moon," Acta Math. 8, 1-36.

Hu, H. Y. \& Wang, Z. H. [2002] Dynamics of Controlled Mechanical Systems with Delayed Feedback (Springer, NY).

Hurwitz, A. [1895] "Über die Bedingungen unter welchen eine Gleichug nur Wurzeln mit negativen reellen Teilen besitz," Math. Ann. 46, 74-81.

Inamura, T. \& Sata, T. [1974] "Stability analysis of cutting under varying spindle speed," Ann. CIRP 23, $119-120$

Ince, E. L. [1956] Ordinary Differential Equations (Dover Publications Inc., NY).

Insperger, T. \& Stépán, G. [2002a] "Stability chart for the delayed Mathieu equation," Proc. Roy. Soc. London A458, 1989-1998.

Insperger, T. \& Stépán, G. [2002b] "Semi-discretization method for general delayed systems," Int. J. Numer. Meth. Engin. 55, 503-518.

Insperger, T. \& Stépán, G. [2003] "Stability of the damped Mathieu equation with time delay," ASME J. Dyn. Syst. Measur. Contr. 125, 166-171.

Insperger, T., Mann, B. P., Stépán, G. \& Bayly, P. V. [2003a] "Stability of up-milling and down-milling, Part 1: Alternative analytical methods," Int. J. Mach. Tools Manuf. 43, 25-34.

Insperger, T., Stépán, G., Bayly, P. V. \& Mann, B. P. [2003b] "Multiple chatter frequencies in milling processes," J. Sound Vibr. 262, 333-345.
Insperger, T., Lekien, F., Salman, H., Haller, G. \& Stépán, G. [2004] "Control of separation point in periodic flows including delay effects," in Proc. IFAC Workshop on Periodic in Control Systems, Yokohama, Japan.

Insperger, T. \& Stépán, G. [2004a] "Updated semidiscretization method for periodic delay-differential equations with discrete delay," Int. J. Numer. Meth. Engin. 61, 117-141.

Insperger, T. \& Stépán, G. [2004b] "Stability analysis of turning with periodic spindle speed modulation via semi-discretization," J. Vibr. Contr. 10, 1835-1855.

Insperger, T. \& Stépán, G. [2004c] "Vibration frequencies in high-speed milling processes, or a positive answer to Davies, Pratt, Dutterer and Burn," ASME J. Manuf. Sci. Engin. 126, 481-487.

Insperger, T. \& Stépán, G. [2004d] "Stability improvements of robot control by periodic variation of the gain parameters," in Proc. 11th World Congress in Mechanism and Machine Science, ed. Huang, T., Tianjin, China (China Machinery Press), pp. 18161820.

Kalmár-Nagy, T., Stépán, G. \& Moon, F. C. [2001] "Subcritical Hopf bifurcation in the delay equation model for machine tool vibrations," Nonlin. Dyn. 26, 121-142.

Kolmanovskii, V. B. \& Nosov, V. R. [1986] Stability of Functional Differential Equations (Academic Press, London).

Leine, R. I., van Campen, D. H. \& van de Vrande, B. L. [2000] "Bifurcations in nonlinear discontinuous systems," Nonlin. Dyn. 23, 105-164.

Levi, M. \& Broer, H. [1995] "Geometrical aspects of stability theory for Hill's equations," Arch. Rat. Mech. Anal. 131, 225-240.

Mann, B. P., Insperger, T., Bayly, P. V. \& Stépán, G. [2003] "Stability of up-milling and down-milling, Part 2: Experimental verification," Int J. Mach. Tools Manuf. 43, 35-40.

Mathieu, E. [1868] "Mémoire sur le mouvement vibratoire d'une membrane de forme elliptique," J. Math. Pure Appl. 13, 137-203.

Minis, I. \& Yanushevsky, R. [1993] "A new theoretical approach for the prediction of machine tool chatter in milling," J. Engin. Indust. 115, 1-8.

Minorsky, N. [1942] "Self-excited oscillations in dynamical systems possessing retarded actions," ASME J. Appl. Mech. 9, 65-72.

Myshkis, A. D. [1949] "General theory of differential equations with delay," Uspehi Mat. Nauk. 4, 99-141; Engl. Transl. [1951] AMS, 55, pp. 1-62.

Neimark, J. I. [1949] "D-subdivision and spaces of quasipolynomials," Prikladnaja Mathematika i Mechanika 13, 349-380.

Niculescu, S. I. [2000] Delay Effects on Stability: A Robust Control Approach (Springer, NY). 
Olgac, N. \& Sipahi, R. [2002] "An exact method for the stability analysis of time delayed LTI Systems," IEEE Trans. Autom. Contr. 47, 793-797.

Peigne, G., Paris, H., Brissaud, D. \& Gouskov, A. [2004] "Impact of the cutting dynamics of small radial immersion milling operations on machined surface roughness," Int. J. Mach. Tools Manuf. 44, 11331142.

Pontryagin, L. S. [1942] "On the zeros of some elementary transcendental functions (in Russian)," Izv. Akad. Nauk SSSR 6, 115-134.

Porter, B. [1967] Stability Criteria for Linear Dynamical Systems (Oliver and Boyd, Edinburgh-London).

Raibert, M. H. \& Craig, J. J. [1981] "Hybrid position force control for computer controlled manipulators," ASME J. Dyn. Syst. Measur. Contr. 102, 125-133.

Rayleigh (Strutt) J. W. [1887] "On the maintenance of vibrations by forces of double frequency, and on the propagation of waves through a medium endowed with a periodic structure," Philos. Mag. J. Sci. 24, $145-159$.

Routh, E. J. [1877] "A treatise on the stability of a given state of motion," London.

Schlippe, B. \& v. Dietrich, R. [1941] "Shimmying of a pneumatic wheel," in Lilienthal-Gesell-Schaft für Luftfahrtforschung, Bericht 140, 125-160 (translated for the AAF in 1947 by Meyer \& Company).

Stépán, G. [1989] Retarded Dynamical Systems (Longman, Harlow).

Stépán, G., Steven, A. \& Maunder, L. [1990] "Design principles of digitally controlled robots," Mech. Mach. Th. 25, 515-527.

Stépán, G. \& Kalmár-Nagy, T. [1997] "Nonlinear regenerative machine tool vibration," Proc. 17th Biennial Conf. Mechanical Vibration and Noise, ASME Design Engineering Technical Conf. (Sacramento) DETC97/VIB-4021, 1-11.

Stépán, G. [1998] "Delay-differential equation models for machine tool chatter," in Nonlinear Dynamics of Material Processing and Manufacturing, ed. Moon, F. C. (John Wiley, NY), pp. 165-192.

Stépán, G. [2001a] "Vibrations of machines subjected to digital force control," Int. J. Solids and Structures 38, 2149-2159.
Stépán, G. [2001b] "Modelling non-linear regenerative effects in metal cutting," Philos. Trans. Roy. Soc. 359, 739-757.

Stépán, G., Szalai, R., Mann, B., Bayly, P., Insperger, T., Gradisek, J. \& Govekar, E. [2004] "Nonlinear dynamics of high-speed milling — analyses, numerics and experiments," ASME J. Vibr. Acoust., in press.

Stephenson, A. [1908] "On a new type of dynamical stability," Mem. Proc. Manch. Lit. Phil. Soc. 52, $1-10$.

Szalai, R. [2002] "Nonlinear vibrations of interrupted cutting processes," MSc thesis, Budapest University of Technology and Economics.

Szalai, R. \& Stépán, G. [2003] "Stability boundaries of high-speed milling corresponding to period doubling are essentially closed curves," in Proc. 2003 ASME Int. Mechanical Engineering Congress and R\&D Expo (Washington D.C.), CD-ROM: IMECE2003-42122, pp. $1-6$.

Szalai, R., Stépán, G. \& Hogan, S. J. [2004] "Global dynamics of low immersion high-speed milling," Chaos 14, 1069-1077.

Takemura, T., Kitamura, T., Hoshi, T. \& Okushima, K. [1974] "Active suppression of chatter by programmed variation of spindle speed," Ann. CIRP 23, 121122 .

Tlusty, J., Polacek, A., Danek, C. \& Spacek, J. [1962] Selbsterregte Schwingungen an Werkzeugmaschinen (VEB Verlag Technik, Berlin).

Tlusty, J. [2000] Manufacturing Processes and Equipment (Prentice Hall, NJ).

Tobias, S. A. [1965] Machine Tool Vibrations (Blackie, London).

van der Pol, F. \& Strutt, M. J. O. [1928] "On the stability of the solutions of Mathieu's equation," Philos. Mag. J. Sci. 5, 18-38.

Volterra, V. [1928] "Sur la theorie mathematique des phenomenes hereditares," J. Math. Pures et Appl. 7, 149-192.

Whitney, D. E. [1977] "Force feedback control of manipulator fine motions," ASME J. Dyn. Syst. Measur. Contr. 98, 91-97. 